\title{
Clinical outcomes in men with prostate cancer who selected active surveillance using a clinical cell cycle risk score
}

\author{
Sanjeev Kaul ${ }^{\ddagger}$, Kirk J Wojno ${ }^{\ddagger 1}$, Steven Stone², Brent Evans², Ryan Bernhisel2 ${ }^{2}$ Stephanie \\ Meek $^{2}$, Richard E D'Anna ${ }^{3}$, Jeffrey Ferguson ${ }^{4}$, Jeffrey Glaser ${ }^{5}$, Todd M Morgan ${ }^{6}$, Jeremy \\ Lieb7$^{7}$, Robert Yan ${ }^{8}$, Todd Cohen ${ }^{9}$ \& Behfar Ehdaie*,10 \\ ${ }^{1}$ Comprehensive Urology, Royal Oak, MI 48073, USA \\ ${ }^{2}$ Myriad Genetics, Inc., Salt Lake City, UT 84108, USA \\ ${ }^{3}$ Arkansas Urology, Little Rock, AR 72211, USA \\ ${ }^{4}$ Urological Associates, Colorado Springs, CO 80907, USA \\ ${ }^{5}$ Specialists in Urology, LLC, Lake Saint Louis, MO 63367, USA \\ ${ }^{6}$ Department of Urology, University of Michigan, Ann Arbor, MI 48109, USA \\ ${ }^{7}$ Pacific Urology, Concord, CA 94520, USA \\ ${ }^{8}$ Golden Gate Urology, San Francisco, CA 94107, USA \\ ${ }^{9}$ Myriad Genetic Laboratories, Inc., Salt Lake City, UT 84108, USA \\ ${ }^{10}$ Memorial Sloan Kettering Cancer Center, New York, NY 10065, USA \\ *Author for correspondence: Tel.: +1 646422 4406; ehdaieb@mskcc.org \\ $¥$ Authors contributed equally
}

\begin{abstract}
Aim: To evaluate active surveillance (AS) selection, safety and durability among men with low-risk prostate cancer assessed using the clinical cell cycle risk (CCR) score, a combined clinical and molecular score. Patients \& methods: Initial treatment selection (AS vs treatment) and duration of AS were evaluated for men with low-risk prostate cancer according to the CCR score and National Comprehensive Cancer Network guidelines. Adverse events included biochemical recurrence and metastasis. Results: $82.4 \%$ (547/664) of men initially selected AS (median follow-up: 2.2 years), $0.4 \%(2 / 547)$ of whom experienced an adverse event. Two-thirds of patients remained on AS for more than 3 years; patient choice was the most common reason for leaving AS. Conclusion: The CCR score may aid in the identification of men who can safely defer prostate cancer treatment.
\end{abstract}

First draft submitted: 17 July 2019; Accepted for publication: 19 August 2019; Published online:

4 September 2019

Keywords: active surveillance $\bullet$ molecular score $\bullet$ prostate cancer $\bullet$ risk assessment $\bullet$ survival

Prostate cancer is the most commonly diagnosed cancer among men in the developed world [1]. Despite this, the majority of men who develop prostate cancer will not die from their disease. Many prostate cancers will remain indolent and not progress to cause significant morbidity or mortality. Deferring treatment through active surveillance (AS) of such patients may be an appropriate alternative to immediate treatment. Urologists and radiation oncologists must therefore weigh oncologic factors, potential treatment-related morbidities and patient preferences to help guide initial management decisions between immediate treatment and AS. Long-term survival in patients placed on AS has been shown to be as high as $98 \%$ for up to 10 years of follow-up, and $94 \%$ up to 15 years of follow-up [2,3]. As a result, AS is now considered standard of care for all men with very low-risk prostate cancer and most men with low-risk prostate cancer according to both the American Urological Association and the National Comprehensive Cancer Network (NCCN) guidelines [4,5].

Currently, selection of AS for men with prostate cancer relies primarily on risk assessment using standard clinicopathologic measures, including prostate-specific antigen (PSA) levels, digital rectal exam and Gleason score. However, risk assessment using these criteria alone has resulted in low AS selection rates, even among men with low-risk disease [6,7]. While adoption of AS has increased during recent years, the AS selection rate for clinically low-risk patients remains between 40 and 50\%, resulting in overtreatment of many men with low-risk disease [8,9]. 
Previous studies have demonstrated that the clinical cell cycle risk (CCR) score, a combined clinical and molecular score, provides significantly improved prognostic information and risk assessment compared with clinicopathologic parameters alone [10-12]. More recently, a CCR score threshold has been developed and validated to facilitate selection of appropriate AS candidates. This AS threshold is defined as having a disease-specific mortality (DSM) of less than or equal to $3.2 \%$ and has been validated in a cohort of conservatively managed men with long-term outcomes [13]. Multiple validation studies have shown that risk classification based on CCR score predicts meaningful long-term, oncologic outcomes more accurately than clinicopathologic features alone [10,12-14]. Therefore, use of the CCR score may improve patient-appropriate treatment selection by reducing risk misclassification and potential over- or undertreatment of patients with prostate cancer.

Many men who initially select AS ultimately transition to definitive treatment, with one study suggesting that approximately a third of men receive definitive treatment within 3 years of diagnosis [15]. While a change in risk classification often prompts this transition, patient anxiety and side effects from repeated biopsies can also prompt a switch to definitive treatment $[15,16]$. Improved risk stratification through the use of validated prognostic tools may increase patient and physician confidence and reduce anxiety around AS, ultimately increasing AS durability and avoiding unnecessary intervention. This study sought to evaluate the selection, safety and durability of AS among men newly diagnosed with prostate cancer who received a CCR score.

\section{Materials \& methods \\ Cohort}

This multicenter, retrospective, observational study included men diagnosed with localized prostate cancer between January 2013 and August 2017 who previously received cell cycle progression (CCP) testing during the course of routine care (Myriad Genetic Laboratories, Inc., UT, USA). Data for patients who had low-risk disease according to both the CCR score (DSM $\leq 3.2 \%$ ) [13] and NCCN guidelines [17] were recorded by urologists from eight community and academic sites (Arkansas Urology [AR, USA]; Comprehensive Urology [MI, USA]; Golden Gate Urology [CA, USA]; Memorial Sloan Kettering Cancer Center [NY, USA]; Pacific Urology [CA, USA]; Specialists in Urology, LLC [MO, USA]; University of Michigan [MI, USA] and Urological Associates, PC [CO, USA]). Patients were excluded if they received definitive therapy prior to diagnostic biopsy, had a life-expectancy of less than 10 years, had a history of hypogonadism or had another malignancy (excluding nonmelanoma skin cancer). Clinical data for each patient were obtained from provider-completed test request forms and through retrospective chart review. AS protocols were left to the discretion of the treating urologist, with pertinent data appropriately documented, including subsequent biopsies, selection of definitive treatment and reasons for both. All AS patients were monitored according to institutional protocols. Typically, sites had patient follow-up 6 months after diagnosis and most had annual follow-up after that. For some sites, follow-up occurred on a semiannual basis. Followup consisted of a combination of PSA, digital rectal exam, biopsy and/or MRI, depending on the institution's protocol. Any disease progression, including biochemical recurrence (BCR), metastasis or death from any cause (cause specified), was documented. Pathology review was directed by dedicated genitourinary pathologists at each institution.

This study was conducted with Institutional Review Board oversight (Quorum, identifier 33138; MSKCC and UM internal Institutional Review Boards). This study involved anonymized, retrospective chart review and patient consent was waived. This study was supported by Myriad Genetics, Inc. and the authors from the funding institution contributed to study design, analysis and reporting.

\section{CCP testing}

Testing was performed as described previously $[10,18,19]$ in a Clinical Laboratory Improvement Amendmentsand College of American Pathology-approved commercial testing laboratory (Myriad Genetic Laboratories, Inc.). Briefly, 1-5 slides containing 5- $\mu \mathrm{m}$ thick sections of formalin-fixed, paraffin-embedded prostate biopsy tissue were macro-dissected as directed by the on-site pathologist. After deparaffinization, total RNA was extracted using a miRNeasy kit (Qiagen) according to manufacturer's instructions and converted to single-strand cDNA. Expression data for 31 CCP genes and 15 housekeeper genes for normalization were generated via TaqMan Low Density arrays in triplicate (Applied Biosystems) [18,19]. A molecular CCP score was calculated as the normalized expression of CCP genes (average of the three replicates minus the average of the appropriate housekeeper genes). UCSF Cancer of the Prostate Risk Assessment (CAPRA) scores [20,21] were calculated using clinical information from provider-completed test request forms as previously described [20]. The CCR score was calculated as a combination 
of the molecular $(\mathrm{CCP})$ and clinical (CAPRA) scores $(0.39 \times \mathrm{CCP}+0.57 \times$ CAPRA) [10]. Estimated DSM based on the CCR score was reported, and the AS threshold (DSM $\leq 3.2 \%$, i.e., low-risk) was indicated for tests reported after 25 September 2015.

\section{Statistical analysis}

Statistical analyses were performed according to a prespecified analysis plan using SAS ${ }^{\circledR}$ software (SAS Institute, Inc., NC, USA) and R software (R Foundation for Statistical Computing, Vienna, Austria). The evaluable study cohort included all patients who passed study inclusion and exclusion criteria. Men were considered as being on AS if they received no definitive treatment within 6 months of diagnosis, and AS duration was defined as the time from diagnosis to first treatment. Clinical outcomes included BCR or metastasis. BCR was defined as PSA greater than $0.2 \mathrm{ng} / \mathrm{ml}$ on $\geq 2$ occasions more than 2 weeks apart following surgery, postexternal beam radiation therapy PSA of nadir $+2 \mathrm{ng} / \mathrm{ml}$ (Phoenix criteria 1 ), or initiation of any salvage therapy $\geq 6$ months after surgery or radiation. Metastasis was confirmed by radiographic evidence.

The Kaplan-Meier method was used to estimate event-free survival (EFS) and time-to-end of AS with $95 \%$ CIs. For primary clinical outcomes, occurrence of BCR or metastasis was considered an event, while initiation of definitive therapy was considered an event in regard to AS durability. Differences in demographic and clinical characteristics between cohorts were tested using the Wilcoxon Rank Sum test of medians for continuous variables and the Fisher's exact test for categorical variables. Missing data were not imputed.

\section{Results}

\section{Cohort}

A total of 664 men with low-risk disease according to both the CCR score and NCCN guidelines were included in the analysis. The patient demographics are summarized in Table 1 . In total, 82.4\% (547/664) of patients selected AS, while 17.6\% (117/664) received definitive treatment within 6 months of diagnosis (Table 1). There were no substantial differences between these groups except for markers of tumor aggressiveness, with men who received definitive treatment within the first 6 months postdiagnosis exhibiting more aggressive pathologic features compared with men who selected AS. Compared with those who selected AS, a higher proportion of men who initially pursued definitive therapy had stage T1c or T2a cancers $(92.3$ vs $74.8 \%$; $<0.001)$ or more than two positive cores ( 47.9 vs $32.9 \%$; $\mathrm{p}=0.003$ ). Across all patients, median CCR score was -0.04 (interquartile range [IQR]: -0.29, 0.27; Table 1), with the full score distribution shown in Supplementary Figure 1.

\section{Survival}

The median follow-up period from time of biopsy for all patients was 2.2 years (IQR: 1.4, 3.0). Five men (0.8\%; 5/664) experienced disease progression events, with two exhibiting metastasis and three showing evidence of BCR following primary therapy. Five-year EFS was 97.8\% (95\% CI: 93.1\%, 99.3\%; Figure 1A) among all patients. Among men who selected AS, the median follow-up time was also 2.2 years (IQR: 1.5, 3.1), with a five-year EFS of 98.5\% (95\% CI: 91.7\%, 99.7\%; Figure 1B). Only two men $(0.4 \% ; 2 / 547)$ who initially selected AS experienced an event. One of these men had Gleason $3+4$ prostate cancer with negative lymph nodes at the time of diagnosis. He had radical prostatectomy after 2.1 years and showed evidence of BCR 3.7 years after diagnosis. A positive surgical margin was present after radical prostatectomy, and it is therefore clinically possible that this case of BCR is related to the remaining positive margins. The second patient who experienced an event was diagnosed with Gleason $3+3$ prostate cancer. While on AS, this patient displayed rising PSA levels and a subsequent biopsy was performed. The disease was upgraded to Gleason $4+4$ postbiopsy and eventually classified as metastatic based on MRI 0.9 years postdiagnosis.

\section{Durability of AS}

Among the 547 men who selected AS, 24.3\% (133/547) received treatment during the course of this study. The vast majority of these men were treated with either radical prostatectomy $(60.9 \%)$ or radiation therapy $(26.3 \%)$. In total, $23.3 \%$ (31/133) of patients who left AS received a definitive treatment due to an increase in Gleason score (Table 2). A number of other factors related to clinical disease progression were also cited as reasons for leaving AS, such as change in PSA and increase in tumor volume (Table 2). The most common reason for leaving AS was 


\begin{tabular}{|c|c|c|c|c|c|}
\hline Characteristic & Statistic/category & AS cohort $(n=547)$ & Treated cohort $(n=117)$ & p-value & Total $(n=664)$ \\
\hline \multirow[t]{2}{*}{ Age at diagnosis (years) } & Mean (SD) & $63.0(7.49)$ & $62.5(8.05)$ & $0.897 \S$ & $62.9(7.59)$ \\
\hline & Min, $\max$ & 41,79 & 39,79 & & 39,79 \\
\hline \multirow[t]{5}{*}{ Race, n (\%) } & White/non-Hispanic & $370(67.6 \%)$ & $80(68.4 \%)$ & $0.147 \mathbb{I}$ & $450(67.8 \%)$ \\
\hline & Black/African & $41(7.5 \%)$ & $14(12.0 \%)$ & & $55(8.3 \%)$ \\
\hline & Hispanic/Latino & $1(0.2 \%)$ & $1(0.9 \%)$ & & $2(0.3 \%)$ \\
\hline & Other & $2(0.4 \%)$ & 0 & & $2(0.3 \%)$ \\
\hline & Unknown ${ }^{\dagger}$ & $129(23.6 \%)$ & $20(17.1 \%)$ & & $149(22.4 \%)$ \\
\hline \multirow[t]{2}{*}{ Prebiopsy PSA (ng/ml) } & Mean (SD) & $5.0(1.86)$ & $5.1(2.02)$ & $0.263^{\S}$ & $5.0(1.89)$ \\
\hline & Median (IQR) & $4.8(3.9,6.0)$ & $5.1(3.8,6.4)$ & & $4.9(3.9,6.1)$ \\
\hline \multirow{3}{*}{ Clinical tumor stage } & T1c & $367(67.1 \%)$ & $95(81.2 \%)$ & & $462(69.6 \%)$ \\
\hline & $\mathrm{T} 2 \mathrm{a}$ & $42(7.7 \%)$ & $13(11.1 \%)$ & & $55(8.3 \%)$ \\
\hline & Missing & $4(0.7 \%)$ & 0 & & $4(0.6 \%)$ \\
\hline \multirow{3}{*}{$\begin{array}{l}\text { Percent positive cores } \\
(\%)^{\ddagger}\end{array}$} & Mean (SD) & $23.0(20.33)$ & $26.2(18.66)$ & $0.010^{\S}$ & $23.5(20.07)$ \\
\hline & Median (IQR) & $16.7(8.3,25.0)$ & $20.1(10.8,33.3)$ & & $16.7(8.3,29.4)$ \\
\hline & Min, $\max$ & $4.2,100$ & $4.2,100$ & & $4.2,100$ \\
\hline \multirow[t]{3}{*}{ Number of positive cores } & $\leq 2$ & $366(66.9 \%)$ & $60(51.3 \%)$ & $0.003 \mathbb{I}$ & $426(64.2 \%)$ \\
\hline & $>2$ & $180(32.9 \%)$ & $56(47.9 \%)$ & & $236(35.5 \%)$ \\
\hline & Missing & $1(0.2 \%)$ & $1(0.9 \%)$ & & $2(0.3 \%)$ \\
\hline CCR score & Mean (SD) & $-0.05(0.39)$ & $0.07(0.41)$ & $0.024 \S$ & $-0.03(0.39)$ \\
\hline
\end{tabular}

\begin{tabular}{|c|c|c|c|}
\hline Reason for leaving AS & $\mathrm{n}$ & $\%$ of patients in AS cohort $(n=547)$ & $\%$ of patients who left AS $(n=133)$ \\
\hline Gleason score & 31 & 5.7 & 23.3 \\
\hline Imaging & 6 & 1.1 & 4.5 \\
\hline Tumor volume & 3 & 0.5 & 2.3 \\
\hline Patient choice & 36 & 6.6 & 27.1 \\
\hline
\end{tabular}

patient choice, accounting for $27.1 \%$ of men who switched from AS to a definitive therapy. In total, $91.2 \%(95 \%$ CI: $88.4 \%, 93.3 \%$ ) of men remained on AS at year 1 (Figure $2 \&$ Table 3). Although this decreased over time, 69.6\% (95\% CI: 64.5\%, 74.1\%) of men who initially selected AS remained on AS at year 3, and 65.2\% (95\% CI: $58.9 \%, 70.8 \%)$ of men remained on AS 4 years postdiagnosis. While the 5-year durability of AS was 50.3\% (95\% CI: $34.6 \%, 64.1 \%$ ), there was only one evaluable patient at year 5, which limits the statistical power. 
(A)

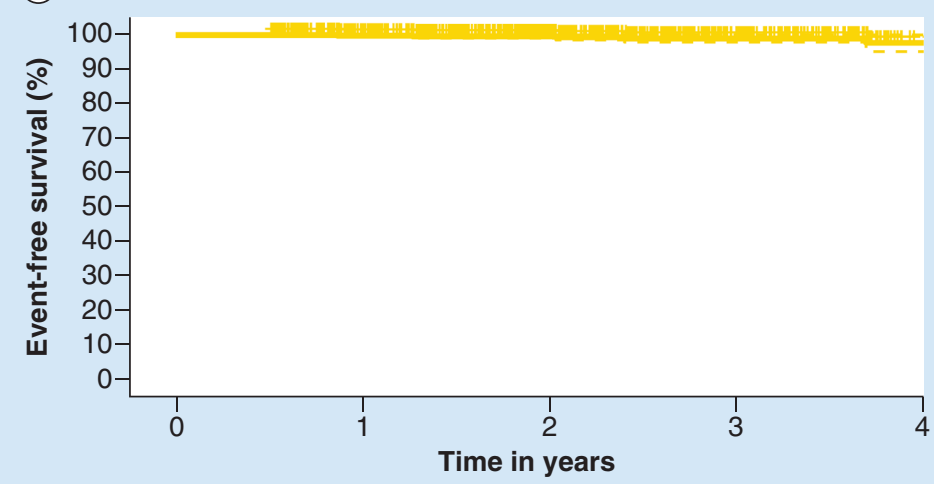

$\begin{array}{lllll}N \text { at risk } & 664 & 570 & 362 & 172\end{array}$

(B)

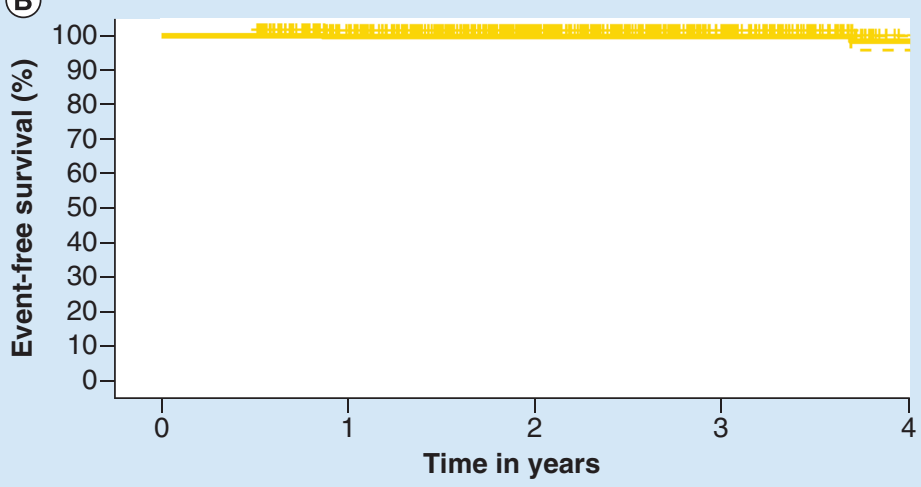

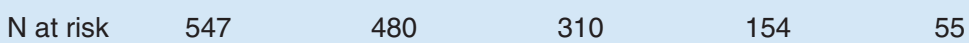

Figure 1. Kaplan-Meier curve for event-free surivival. (A) In the full cohort $(n=664)$ and (B) in the AS cohort $(n=$ 547). $95 \% \mathrm{Cls}$ are shown with the dotted lines.

AS: Active surveillance.

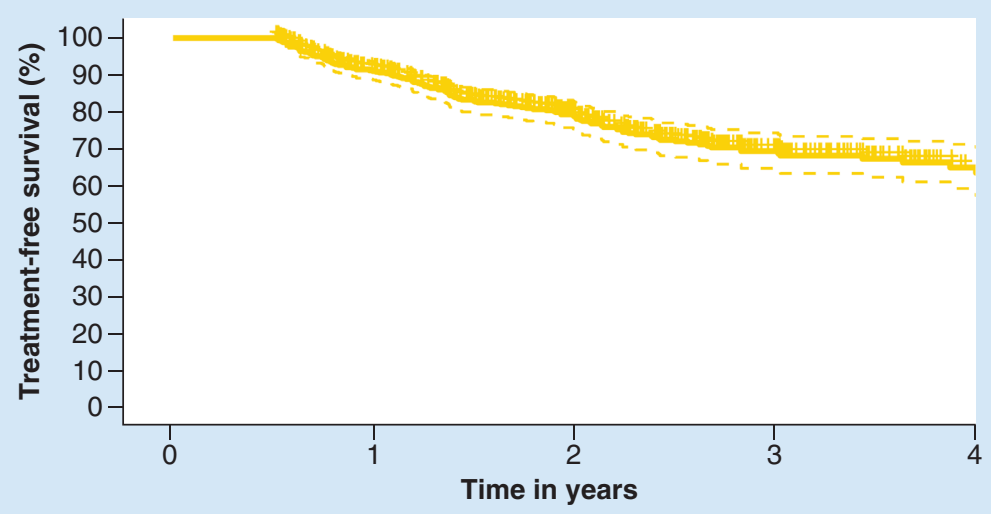

$\begin{array}{lllll}N \text { at risk } & 547 & 441 & 260 & 123\end{array}$

Figure 2. Kaplan-Meier curve for the durability of active surveillance before definitive treatment. $95 \% \mathrm{Cls}$ are shown with the dotted lines. 
Table 3. Kaplan-Meier estimates for the durability of active surveillance $(n=547)$.

\begin{tabular}{|lll|}
\hline Year & Evaluable men $(\mathbf{n})$ & Treatment-free survival (95\% Cl) \\
\hline Year 1 & 441 & $91.2 \%(88.4 \%, 93.3 \%)$ \\
\hline Year 2 & 260 & $78.9 \%(74.8 \%, 82.4 \%)$ \\
\hline Year 3 & 123 & $69.6 \%(64.5 \%, 74.1 \%)$ \\
\hline Year 4 & 45 & $65.2 \%(58.9 \%, 70.8 \%)$ \\
\hline Year 5 & $1 \dagger$ & $50.3 \%(34.6 \%, 64.1 \%)$ \\
\hline
\end{tabular}

† While there was only one evaluable patient at the 5 -year time point, there were evaluable patients between years 4 and 5 who left active surveillance for definitive treatment prior to 5 years. These individuals were used to calculate the treatment-free survival beyond 4 years.

\section{Discussion}

Men with low-risk prostate cancer are increasingly selecting surveillance as their initial management strategy. National trends show that approximately $30-40 \%$ of patients who initially select AS eventually undergo definitive treatment [22]. The most common reasons cited for leaving AS for definitive therapy are clinical progression of the disease and anxiety on the part of the patient and/or physician due to the possibility that the disease may be more aggressive than predicted $[2,15,23]$. The use of a combined clinical and molecular score (CCR) has been shown to provide significantly improved prognostic information over clinicopathologic factors alone [10,11]. As such, the utility of the CCR score was evaluated here as a method to safely improve the identification of men for AS.

In this study, we observed that $82.4 \%$ of men with prostate cancer determined to have a low estimated risk of DSM by both the CCR score and NCCN guidelines initially selected AS. This indicates that most patients with molecularly confirmed low-risk disease and their physicians selected AS for initial clinical management in both community and academic clinical settings. The rate of AS selection in men with low-risk disease classified without the additional prognostic information from molecular testing appears to be lower than the rates observed in this study. The National Cancer Database reports that $20-24 \%$ of men determined to have low-risk prostate cancer by clinicopathologic parameters alone select AS. Underutilization of AS is also observed in the Surveillance, Epidemiology and End Results Program database, the UCSF Cancer of the Prostate Strategic Urologic Research Endeavor registry and the American Urological Association Quality Registry, which report only 30-40\% utilization of AS among men with low-risk disease [24,25]. Although utilization of AS has improved in recent years, the AS rate reported here was substantially higher than in recent studies where only clinicopathologic features were used in decision-making $[6,9,26]$. Importantly, AS selection here was also durable, with most patients maintaining AS for 3 or more years.

Among men with low-risk disease who initially selected AS, only two experienced metastasis or BCR during the study period, which corresponds with a 5-year EFS of 98.5\% for men who selected AS. As with any tumor-based test, sampling error is an inherent limitation of testing. Sampling error when initially testing these tumors could have resulted in an inaccurate risk classification for patients who experienced progressive disease while on AS. This is a common limitation to tests of this class that also impacts the accuracy of clinicopathologic evaluation [27-29], and the extensive validation of the CCP test has previously shown that, despite the potential for sampling error, the CCR score adds accurate information beyond standard clinical features to inform risk of disease progression among men with prostate cancer. These data suggest that selection of AS using the CCR score is initially safe, though longer follow-up of this patient cohort is required to verify these findings. This is comparable to a recently published single-site study by Nyame et al., which showed a 5-year EFS of $97.7 \%$ for patients with NCCN very low/low-risk disease who initially selected AS [30]. In both studies, NCCN criteria were used to select patients for AS, while the study presented here has molecularly confirmed low-risk disease. The present study shows that EFS for low-risk patients is high even across multiple sites.

The durability of AS was similar to previously published rates, with approximately two-thirds of patients remaining on AS after 4 years [30]; however, the increased AS selection rate at the time of diagnosis reported here increased the overall number of men who remained on AS. Among men who left AS, 27.1\% cited patient choice as the reason for leaving. Based on previous studies, this may be partly related to patient anxiety [15]. Most other patients who left AS cited a change in clinicopathologic features. However, aside from the men who experienced an increase in Gleason score or an increase in PSA greater than $10 \mathrm{ng} / \mathrm{ml}$, these changes may not actually represent disease progression. For patients who experienced a clinically insignificant change in clinicopathologic features, anxiety on the part of the patient and/or physician was likely the driving force in leaving AS [15,16]. 
Interestingly, behaviors regarding AS were different among providers and patients at academic institutions $(\mathrm{n}=181)$ as compared with those at community practices $(\mathrm{n}=483)$. Notably, patients treated at academic institutions were significantly more likely to be placed on AS than those in community practices $(92.8$ and $78.5 \%$, respectively; $\mathrm{p}<0.0001$ ), though the durability of AS was not different based on site type (4-year durability academic and community: 69.4\% [95\% CI: 59.4\%, 77.4\%] and 62.8\% [95\% CI: 54.6\%, 69.9\%], respectively; $\mathrm{p}=0.5383$ ). The clinical significance of such a difference cannot be determined from this study. Increased utilization of AS at academic sites could be due to a number of factors including increased comfort with AS as a treatment option and increased comfort and/or familiarity with the CCR score. Differences between institutional protocols for selecting AS may also contribute to this disparity.

This study has some limitations. This study did not include a control arm to evaluate AS selection and durability among men who did not receive a CCR score. However, there is a breadth of available literature that includes men with low-risk disease treated in both academic and community settings over the last 10 years. This offers an informative standard against which AS utilization in this study can be compared. In addition, the median follow-up time reported here is shorter than in similar studies, with a limited proportion of the cohort having follow-up beyond 3 years. Extended observation of this cohort is required to confirm the conclusions regarding safety of the CCR AS threshold over longer time periods. Finally, this cohort is restricted to patients meeting NCCN low-risk criteria and therefore does not include assessment of patients with NCCN favorable-intermediate risk disease who may be candidates for AS accordign to the CCR score.

\section{Conclusion}

Collectively, the data presented here suggest that using the CCR score in prostate cancer risk assessment can safely increase the selection of AS among appropriate men, compared with clinicopathologic criteria alone. Men with molecularly confirmed low-risk disease frequently selected AS in both community and academic clinical practices, tended to remain adherent on AS after initial selection, and their incidence of disease progression was extremely low. Molecular tumor testing is associated with higher costs at the time of initial risk assessment than other standard risk assessment protocols [31]. However, increasing the pool of men who defer costly definitive treatment(s) and maintaining that pool of patients for longer ultimately and meaningfully reduces treatment-associated costs for men with low-risk prostate cancer [31-33]. Overall, utilizing the CCR score during risk assessment may allow more patients to avoid unnecessary overtreatment, thus reducing the financial and physical costs related to treatment of prostate cancer and treatment-related morbidities.

\section{Summary points}

- It may be appropriate to defer definitive treatment for some men with low-risk prostate cancer, according to current active surveillance (AS) guidelines based on low-risk clinical features.

- The clinical cell cycle risk (CCR) score has been validated to identify men with low-risk prostate cancer and provides improved prognostic information over clinical features alone.

- Overall, $82.4 \%$ of men in this cohort with National Comprehensive Cancer Network low-risk prostate cancer and low CCR scores selected AS for their initial treatment.

- Among men who initially went on AS, only $0.4 \%$ experienced disease progression to date.

- At 3 years, $69.6 \%(95 \% \mathrm{Cl}: 64.5 \%, 74.1 \%)$ of men who initially selected AS remained on AS.

- A total of $24.3 \%$ of men who initially went on AS received definitive treatment during the study period, with patient choice being the most commonly cited reason.

- Utilizing the CCR score during risk assessment may allow more patients to safely avoid unnecessary overtreatment, thus reducing the financial and physical costs related to treatment of prostate cancer and treatment-related morbidities.

Supplementary data

To view the supplementary data that accompany this paper please visit the journal website at: www.futuremedicine.com/doi/suppl/10.2217/pme-2019-0084 
and served on an advisory board and speaker's bureau for Myriad Genetics, Inc. and Intuitive Surgical; he has also received research funding from Myriad Genetics, Inc. K Wojno has served on advisory boards and/or speaker's bureaus for Myriad Genetics, Inc., Genomic Health, GenomeDx, MDxHealth and Strand Diagnositics; has receive honoraria from Strand Diagnostics; and has received research funding from Myriad Genetics, Genomic Health, Genome Dx, MDxHealth and NeoGenomics Laboratories. J Glaser has received research funding from Myriad Genetics, Inc. T Morgan has served on an advisory board for Myriad Genetics, Inc. and has received research funding from Myriad Genetics, Inc., MDxHealth and GenomeDx. R Yan has received research funding from Myriad Genetics, Inc. B Ehdaie has received honoraria and research funding from and served on an advisory board for Myriad Genetics, Inc., and received research funding from InSightec The authors have no other relevant affiliations or financial involvement with any organization or entity with a financial interest in or financial conflict with the subject matter or materials discussed in the manuscript apart from those disclosed.

The authors acknowledge Krystal Brown and Jennifer Logan for their assistance in preparing the manuscript.

Open access

This work is licensed under the Attribution-NonCommercial-NoDerivatives 4.0 Unported License. To view a copy of this license, visit http://creativecommons.org/licenses/by-nc-nd/4.0/

\section{References}

Papers of special note have been highlighted as: $\bullet$ of interest; $\bullet \bullet$ of considerable interest

1. Jemal A, Bray F, Center MM, Ferlay J, Ward E, Forman D. Global cancer statistics. CA Cancer J. Clin. 61(2), 69-90 (2011).

2. Welty CJ, Cowan JE, Nguyen $\mathrm{H}$ et al. Extended followup and risk factors for disease reclassification in a large active surveillance cohort for localized prostate cancer. J. Urol. 193(3), 807-811 (2015).

3. Klotz L, Vesprini D, Sethukavalan P et al. Long-term follow-up of a large active surveillance cohort of patients with prostate cancer. J. Clin. Oncol. 33(3), 272-277 (2015).

4. Sanda MG, Cadeddu JA, Kirkby E et al. Clinically Localized Prostate Cancer: AUA/ASTRO/SUO Guideline. Part I: Risk Stratification, Shared Decision Making, and Care Options. J. Urol. 199(3), 683-690 (2018).

- These guidelines contain the current recommendations regarding active surveillance (AS) from the American Urologic Association, American Society for Radiation Oncology and the Society of Urologic Oncology.

5. Mohler JL, Lee RJ, Antonarakis ES et al. NCCN Clinical Practice Guidelines in Oncology: Prostate Cancer (Version 4.2019). NCCN Clinical Practice Guidelines in Oncology (2019). https://www.nccn.org/professionals/physician_gls/pdf/prostate.pdf

- These guidelines contain the current recommendations regarding AS from the National Comprehensive Cancer Network.

6. Cooperberg MR, Broering JM, Carroll PR. Time trends and local variation in primary treatment of localized prostate cancer. J. Clin. Oncol. 28(7), 1117-1123 (2010).

7. Aizer AA, Paly JJ, Zietman AL et al. Multidisciplinary care and pursuit of active surveillance in low-risk prostate cancer. J. Clin. Oncol. 30(25), 3071-3076 (2012).

8. Tosoian JJ, Carter HB, Lepor A, Loeb S. Active surveillance for prostate cancer: current evidence and contemporary state of practice. Nat. Rev. Urol. 13, 205 (2016).

- A comprehensive review of the evidence surrounding and practices regarding AS.

9. Womble PR, Montie JE, Ye Z, Linsell SM, Lane BR, Miller DC. Contemporary use of initial active surveillance among men in Michigan with low-risk prostate cancer. Eur. Urol. 67(1), 44-50 (2015).

10. Cuzick J, Stone S, Fisher G et al. Validation of an RNA cell cycle progression score for predicting death from prostate cancer in a conservatively managed needle biopsy cohort. Br. J. Cancer 113(3), 382-389 (2015).

-• Presents the development and validation of the clinical cell cycle risk (CCR) score.

11. Sommariva S, Tarricone R, Lazzeri M, Ricciardi W, Montorsi F. Prognostic value of the cell cycle progression score in patients with prostate cancer: a systematic review and meta-analysis. Eur. Urol. 69(1), 107-115 (2016).

12. Tosoian JJ, Chappidi MR, Bishoff JT et al. Prognostic utility of biopsy-derived cell cycle progression score in patients with National Comprehensive Cancer Network low-risk prostate cancer undergoing radical prostatectomy: implications for treatment guidance. BJU Int. 120(6), 808-814 (2017).

13. Lin DW, Crawford ED, Keane T et al. Identification of men with low-risk biopsy-confirmed prostate cancer as candidates for active surveillance. Urol. Oncol. 36(6), 310.e317-310.e313 (2018).

-• This manuscript details the development and validation of clinical cell cycle risk threshold score to identify candidates for AS.

14. Cooperberg MR, Simko JP, Cowan JE et al. Validation of a cell cycle progression gene panel to improve risk stratification in a contemporary prostatectomy cohort. J. Clin. Oncol. 31(11), 1428-1434 (2013).

15. Latini DM, Hart SL, Knight SJ et al. The relationship between anxiety and time to treatment for patients with prostate cancer on surveillance. J. Urol. 178(3), 826-832 (2007). 
16. Dall'era MA, Albertsen PC, Bangma C et al. Active surveillance for prostate cancer: a systematic review of the literature. Eur. Urol. 62(6), 976-983 (2012).

- $\quad$ Explores the relationship between patient anxiety and AS decisions.

17. NCCN. NCCN Clinical Guidelines in Oncology (NCCN Guidelines $\left.{ }^{\circledR}\right)$ prostate cancer version 2 (2016). https://www.nccn.org/professionals/

18. Cuzick J, Swanson GP, Fisher G et al. Prognostic value of an RNA expression signature derived from cell cycle proliferation genes in patients with prostate cancer: a retrospective study. Lancet Oncol. 12(3), 245-255 (2011).

19. Warf M, Reid J, Brown K et al. Analytical validation of a cell cycle progression signature used as a prognostic marker in prostate cancer. $J$. Mol. Biomark. Diagn. 6, 4 (2015).

20. Cooperberg MR, Pasta DJ, Elkin EP et al. The University of California, San Francisco Cancer of the Prostate Risk Assessment score: a straightforward and reliable preoperative predictor of disease recurrence after radical prostatectomy. J. Urol. 173(6), 1938-1942 (2005).

21. Cooperberg MR, Freedland SJ, Pasta DJ et al. Multiinstitutional validation of the UCSF Cancer of the Prostate Risk Assessment for prediction of recurrence after radical prostatectomy. Cancer 107(10), 2384-2391 (2006).

22. Romero-Otero J, García-Gómez B, Duarte-Ojeda JM et al. Active surveillance for prostate cancer. Int. J. Urol. 23(3), 211-218 (2015).

23. Burnet KL, Parker C, Dearnaley D, Brewin CR, Watson M. Does active surveillance for men with localized prostate cancer carry psychological morbidity? BJU Int. 100(3), 540-543 (2007).

24. Noone AM, Howlander N, Krapcho M et al. SEER cancer statistics review, 1975-2015. National Cancer Institute, MD, USA (2017). https://seer.cancer.gov/archive/csr/1975_2015/

25. Cooperberg MR, Broering JM, Litwin MS et al. The contemporary management of prostate cancer in the United States: lessons from the cancer of the prostate strategic urologic research endeavor (CAPSURE), a national disease registry. J. Urol. 171(4), 1393-1401 (2004).

26. Loeb S, Folkvaljon Y, Makarov DV, Bratt O, Bill-Axelson A, Stattin P. Five-year nationwide follow-up study of active surveillance for prostate cancer. Eur. Urol. 67(2), 233-238 (2015).

27. Nyame YA, Almassi N, Haywood SC et al. Intermediate-term outcomes for men with very low/low and intermediate/high risk prostate cancer managed by active surveillance. J. Urol. 198(3), 591-599 (2017).

28. Bonini P, Plebani M, Ceriotti F, Rubboli F. Errors in laboratory medicine. Clin. Chem. 48(5), 691-698 (2002).

29. Shipitsin M, Small C, Choudhury $S$ et al. Identification of proteomic biomarkers predicting prostate cancer aggressiveness and lethality despite biopsy-sampling error. Br. J. Cancer. 111(6), 1201-1212 (2014).

30. Nyame YA, Almassi N, Haywood SC et al. Intermediate-term outcomes for men with very low/low and intermediate/high risk prostate cancer managed by active surveillance. J. Urol. 198(3), 591-599 (2017).

31. Crawford ED, Cole D, Lewine N, Gustavsen G. Evaluation of the economic impact of the CCP assay in localized prostate cancer. J. Clin. Oncol. 33((7_suppl)), 85 (2015).

32. Shore ND, Kella N, Moran B et al. Impact of the cell cycle progression test on physician and patient treatment selection for localized prostate cancer. J. Urol. 195(3), 612-618 (2016).

33. Eldefrawy A, Katkoori D, Abramowitz M, Soloway MS, Manoharan M. Active surveillance vs. treatment for low-risk prostate cancer: A cost comparison. Urol. Oncol. 31(5), 576-580 (2013). 
